\title{
The Effect of Application Parameter of Pulsed Direct Current on Wound Healing of Patients with Pressure Ulcer
}

\begin{abstract}
This study investigated the effects of changes to the pulsation factor of pulsed direct currents on wound healing. Patients with a pressure ulcer at a care hospital for the elderly were randomly divided into three groups: Group 1 involved the application of $100 \mu \mathrm{s}$ in pulse duration, $10 \mathrm{~ms}$ in pulse period, $100 \mathrm{pps}$ in a pulsation factor, $15 \mathrm{~mA}$ in pulse amplitude, and polarity red+ by using pulsed direct currents; Group 2 involved a change of pulse period to $8 \mathrm{~ms}$; and Group 3 received general wound management. Although there were no statistically significant differences in the changing stages of pressure ulcers among the groups, all the groups dropped in numerical stages. In the two groups to which pulsed direct currents were applied, there was a statistically significant reduction in the stages of pressure ulcers from the initial assessment to the 12-week assessment ( $p$ (.05). Even though there were no statistically significant differences in changes to the area of pressure ulcers among the groups, a statistically significant decrease was found in pulsed direct current group 2 whose pulse period was shortened ( $p<.05)$. There was no difference in the healing rate of pressure ulcers among the groups, but it made a numerical increase in pulsed direct current group 1 and group 2 and a numerical decrease in group 3 . There were no significant differences in the characteristics of those who had a full recovery among the groups. Those findings indicate that pulsed direct currents have positive effects on the wound healing of patients with a pressure ulcer and that a treatment with pulsed direct currents whose pulsation factor is raised by reducing the pulse duration is especially effective.
\end{abstract}

Key words: Pressure Ulser; Wound Healing; Pulsed Drect Current
Ga Yeong Kima, Sang Bin Lee Ok Kon Moon ${ }^{c}$, Ji Sung Kim ${ }^{\text {, }}$, Jung Hyun Choi ${ }^{b}$, Jung San Wang ${ }^{\mathrm{e}}$, Joo Hyun Park ${ }^{d}$, Hong Rae Kim', Ju Hwan Lee ${ }^{g}$, Kyung Ok Min ${ }^{\text {h }}$

aHoward Hill Hospital, Yongin; ${ }^{b}$ Namseoul University, Cheonan; "Howon University, Gunsan; 'Suwon Women's College, Suwon; 'Yeoju Institute of Technology, 'Yeoju; Kyungwoon University, Gumi; 'Veterans Health Service Medical Center, Seoul; ${ }^{\text {hYongin }}$ University, Yongin, Korea

Received : 16 May 2014

Revised : 20 August 2014

Accepted : 02 September 2014

\section{Address for correspondence}

Kyung Ok Min, PT, Ph.D

Department of Physical Therapy, Yongin University, 470 Samga-dong, Cheoingu, Yongin, Korea

Tel: 82-31-8020-2771

E-mail: komin@yongin.ac.kr

\section{INTRODUCTION}

One of the main skin disorders common to all medical facilities accommodating the elderly, pressure ulcers have been on the rise with the increasing numbers of elderly and chronic and critically ill patients around the world. Multilateral efforts have been made to treat pressure ulcers $(1,2)$. Of the elderly patients hospitalized in care hospitals for the elderly, those who have a nervous system disorder show a higher frequency of pressure ulcers by 30 60\% than those who have other disorders, which is because they suffer hemi plegia due to neurological damage and loss of sensory functions and thus are more likely to develop a pressure ulcer $(3,4)$. The possibility rises even further as patients at elderly care facilities have deteriorated in physical functions and mobility than the elderly in the community $(5,6)$. Some previous studies on electrical stimulation for patients with a pressure ulcer reported that direct currents facilitated chemical effects and metabolic activities and thus tissue healing(7) and confirmed that pulsed electrical stimulation of low output strength and high voltage recorded an average of $45 \%$ wound healing per week(8). It was also found that anode further facilitated wound healing in terms of polarity of electric currents and that cathode was more effective for inhibiting inflammation(9). A study on wound healing in rabbits reported that the application of pulsed currents with a pulse frequency of 80 pps resulted in a significant increase of wound tension and mitotic cells and that electrical stimulation 
accelerated epithelization and thus facilitated the wound healing capability(10). A study stimulated pressure ulcers in spinalized patients with anode pulsed currents with a pulse frequency of 100 pps and reported a significant increase in the speed of pressure ulcer healing(11). Such previous studies on electrical stimulation related to tissue healing used animal subjects with a low frequency of studies using human subjects $(12,13)$. There have been many different studies that proved the effects of electrical stimulation on chronic wounds, but the application parameter format of electrotherapy applicable to pressure ulcers has not been determined yet. Some studies pointed to a need to establish a standardized treatment stage for such variables as the amount of electricity and the duration of treatment $(14,15)$. This study thus set out to demonstrate the effects of pulsed direct currents on wound healing by making changes to the pulse period in an electrotherapy group and a standard care treatment group.

\section{METHODS}

\section{Subject}

The subjects include patients aged 65 or older in $\mathbb{I} \sim \mathbb{V}$ of NPUAP-EPUAP (2009) at $\mathrm{H}$ Hospital in Gyeonggi Province. They were not denied a position change due to treatment reasons, had no cardiovascular, internal or orthopedic disease that could affect treatment, suffered no inflammation in the pressure ulcer areas, and understood the purposes of the study and consented to participate in it. In case of unconscious patients, permission was obtained from their caregivers. A total of 34 patients with characteristics appropriate for the study took part in it, and 29 of them stayed with it to the end after those who were transferred to another hospital or underwent surgery according to the progress of pressure ulcer area based on a surgeon's examination were excluded.

Table 1. General and pressure ulcer characteristics of the subjects

\begin{tabular}{cccc} 
& & & \\
& $1(\mathrm{n}=9)$ & $2(\mathrm{n}=9)$ & $3(\mathrm{n}=11)$ \\
\hline Age & $74.78 \pm 5.78$ & $76.89 \pm 5.30$ & $77.18 \pm 7.83$ \\
SA & $2.44 \pm 0.73$ & $2.56 \pm 0.73$ & $2.73 \pm 0.91$ \\
IWSA & $13.11 \pm 8.74$ & $12.67 \pm 7.79$ & $10.70 \pm 18.14$ \\
\hline
\end{tabular}

SA : Stage average

IWSA : Initial wound surface area

\section{Procedure}

The patients that met the selection criteria were randomly assigned to three groups in a 12 -week study. The conditions of Pulsed Direct Current 1 include $100 \mu \mathrm{s}$ of pulse duration, $10 \mathrm{~ms}$ of pulse period, $100 \mathrm{pps}$ of pulsation factor, polarity red+, and $15 \mathrm{~mA}$ of pulse amplitude. Pulsed Direct Current 2 applied 8 $\mathrm{ms}$ of pulse period and $125 \mathrm{pps}$ of pulsation factor three times a week with each treatment lasting 60 minutes. Group 3 received standard care treatment for pressure ulcers.

\section{Measuring Instrument}

The pressure ulcer stages were categorized according to the criteria of NPUAP-EPUAP (2009)(Table 2).

Table 2 Pressure ulcer stage

\begin{tabular}{ll}
\hline \multicolumn{1}{c}{ Stage } \\
\hline Intact skin with non-blanchable erythema of a local- \\
ized area usually over a bony prominence. \\
Discoloration of the skin, warmth, edema, hardness \\
of pain may also be present. \\
Partial thickness loss of dermis presenting as a \\
shallow open ulcer with a red pink wound bed, \\
without slough. May also present as an intact or \\
open/ruptured serum or sero-sanguinous-filled \\
blister. \\
Full thickness skin loss. Subcutaneous fat may be \\
visible but bone, tendon or muscle are not \\
exposed. Some slough may be present. May \\
include undermining and tunneling. \\
Full thickness tissue loss with exposed bone, ten- \\
don or muscle. Slough or eschar may be present. \\
Open includes undermining and tunneling
\end{tabular}

The Ferrel(16) method was used to obtain the wound surface area (WSA) and percentage wound surface area reduction from baseline (\% \ WSA)(17).

\section{Data Analysis}

Collected data were treated with SPSS 18.0. repeated one-way ANOVA was conducted to test significant differences, if any, at the initial assessment and the $4-, 8$, and $12-$ week point among the groups. The statistical significance level $\alpha$ was set at .05. 


\section{RESULTS}

\section{Changes to the NPUAP Stages}

All of the three groups showed a reduction to average changes to the stages of pressure ulcers (Table 3), but there were no statistically significant differences among them. However, there was a statistically significant decrease in average changes between the initial assessment and the 12-week assessment in Pulsed Direct Current 1 and 2 Group ( $p<.05)$ and in stage changes between the initial assessment and the 4-week assessment in Pulsed Direct Current 1 Group (p<.05).

Table 3. The comparison of NPUAP stage

\begin{tabular}{ccccc}
\hline & 1 & 2 & 3 & $p$ \\
\hline pre & $2.44 \pm 0.73$ & $2.56 \pm 0.73$ & $2.73 \pm 0.91$ & .81 \\
$4 w k s$ & $1.67 \pm 1.41^{a}$ & $2.00 \pm 1.32$ & $2.64 \pm 1.21$ & .38 \\
8wks & $1.44 \pm 1.51$ & $1.44 \pm 1.51$ & $2.55 \pm 1.44$ & .22 \\
$12 w k s$ & $1.00 \pm 1.58^{\circ}$ & $1.22 \pm 0.73^{b}$ & $2.18 \pm 0.91$ & .31 \\
\hline
\end{tabular}

a: pre $>4 w k s$, b: pre $>12 w k s$

\section{Changes to WSA}

Table 4 shows changes to WSA. Although there were no statistically significant differences among the groups, there was a statistically significant reduction in WSA between the initial assessment and the 4-week assessment in Pulsed Direct Current 1 Group ( $\mathrm{p}<.05)$ and also in WSA between the initial assessment and the 4-week, between the 4-week and the 8 -week assessment, and between the initial assessment and the 12-week in Pulsed Direct Current 2 Group ( $\mathrm{p}<.05)$.

Table 4. The comparison of wound surface area

\begin{tabular}{ccccc}
\hline & 1 & 2 & 3 & $\mathrm{p}$ \\
\hline pre & $13.11 \pm 8.74$ & $12.66 \pm 7.79$ & $10.80 \pm 18.12$ & .81 \\
$4 \mathrm{wks}$ & $7.33 \pm 6.08$ & $7.89 \pm 7.75$ & $9.50 \pm 16.00$ & .38 \\
$8 \mathrm{wks}$ & $5.22 \pm 5.65$ & $5.44 \pm 5.98$ & $11.05 \pm 16.58$ & .22 \\
$12 \mathrm{wks}$ & $3.00 \pm 4.74$ & $4.11 \pm 6.01$ & $10.82 \pm 16.83$ & .31 \\
\hline
\end{tabular}

a: pre $>4 w k s$, b: pre>12wks

\section{Comparison of Healing Rates of Pressure Ulcers}

Table 5 presents the healing rates of the three groups. There was no statistical significance among them. The average healing rate rose in Groups A and B and dropped in Group C.
Table 5. The comparison of healing rate outcome (\%)

\begin{tabular}{ccccc}
\hline & 1 & 2 & 3 & $p$ \\
\hline $4 w k s$ & $56.42 \pm 39.79$ & $42.72 \pm 40.59$ & $19.14 \pm 34.12$ & .10 \\
8wks & $65.38 \pm 43.70$ & $62.15 \pm 38.32$ & $-41.29 \pm 177.40$ & .08 \\
$12 w k s$ & $78.47 \pm 36.05$ & $75.11 \pm 29.83$ & $-41.29 \pm 194.40$ & .06 \\
\hline
\end{tabular}

\section{Characteristics of Full Recovery Patients of Pressure Ulcers (healing rate $=100 \%$ )}

Table 6 shows the characteristics of patient subjects whose healing rate reached $100 \%$ at the 12 -week point including the age at the time of the initial assessment, stages of pressure ulcers, and WSA at the initial assessment. There were five, five, and four patients that fully recovered from pressure ulcers in Pulsed Direct Current Group 1, Pulsed Direct Current 2 Group, and Standard Care Treatment Group, respectively. There were no significant changes in the age, stage of pressure ulcer, and WSA at the initial assessment among the groups.

Table 6. Characteristics of the complete healing

\begin{tabular}{ccccc}
\multicolumn{3}{c}{ subjects } & \multicolumn{2}{c}{ (Mean \pm SD) } \\
\hline & $1(n=5)$ & $2(n=5)$ & $3(n=4)$ & $p$ \\
\hline age & $75.80 \pm 4.55$ & $78.80 \pm 3.03$ & $75.50 \pm 5.45$ & .68 \\
ISA & $2.20 \pm 0.45$ & $2.20 \pm 0.45$ & $02.00 \pm 0.00$ & .22 \\
IWSA & $14.20 \pm 11.01$ & $10.00 \pm 6.40$ & $03.56 \pm 3.07$ & .46 \\
\hline
\end{tabular}

ISA : Initial stage average

IWSA : Initial wound surface area

\section{DISCUSSION}

The present study aimed to investigate the effects of a physical therapy intervention using pulsed direct currents on clinical results. Groups of Pulsed Direct Current 1 and 2 had the application of the same total pulse duration and amplitude and then a different pulse period at $10 \mathrm{~ms}$ and $8 \mathrm{~ms}$, respectively, to change the pulsation factor. The findings show no significant differences among the groups, but there was a statistically significant decrease in pressure ulcer stages and WSA in Groups 1 and 2, which was in line with Kloth(8) and Feedar(18) that reported the effects of pulsed direct currents and Houghton(17) that found that electrical stimulation accelerated wound healing. As for the healing rate of pressure ulcers, there was a reduction of WSA between the 
initial assessment and the 4-week assessment in all the three groups. At the 8-week point, Pulsed Direct Current Group 1 recorded 65.38 $\pm 43.70 \%$,Pulsed Direct Current Group $262.15 \pm 38.32 \%$, and Group 3 $-41.29 \pm 177.40$, which indicates that Group 3 made an increase in WAS at the 8-week point and grew worse in the healing rate of pressure ulcers. Those findings were in line with Wood(19) reporting that the group of low-strength pulsed direct currents recorded a healing rate of $58 \%$ and that only one of 31 patients reduced in WSA in the control group that received on electrical stimulation with the rest rising in WSA. Yim(3) also reported similar results that the patients with a nervous system disorder and pressure ulcer deteriorated in the pressure ulcer state in $54.6 \%$ and maintained the current pressure ulcer state in $36.4 \%$. Once pressure ulcers occur, it becomes difficult to treat patients and leads to the prolongation of treatment period, which results in the rising percentage of maintenance and deterioration(20). It explains why WSA increased in Group 3 in the present study.

It was not the case that all the patients made a full recovery during the study, but six of nine, five of nine, and four of eleven recorded a 100\% healing rate in Group 1, 2, and 3, respectively, over the 12 weeks, which was in line with Adunsky(7) that confirmed a reduction in WSA through eight weeks of electrical stimulation and 12 weeks of follow-up study and found no significant differences in the full recovery patients among the groups. Given the findings of those previous studies and the present study, it seems that the early state of pressure ulcers influences full recovery. It has been reported that the effects of independent treatments on the reduction of pressure ulcers including cleanliness, dressing, electrical stimulation, and internal elements such as platelets and growth factors are insignificant and that the effects are the greatest when those elements are applied together(21). It is thus required to carry out a variety of interventions including electrical stimulation at an early stage of pressure ulcers in a proper manner(22).

\section{CONCLUSION}

The present study set investigated differences in clinical effects according to the changing pulsation factors of pulsed direct currents with patients with a pressure ulcer and found that the application of pulsed direct currents had positive effects on the early treatment of such patients and that Pulsed Direct Current Group 2 whose pulsation factor was raised by reducing the pulse duration was more effective. The study also found that the full recovery rate of pressure ulcers influenced the early state of pressure ulcers. Based on those findings, the study made a proposal for appropriate research on pressure ulcers through a physical therapy intervention and research on various protocols for the full recovery of patients with a pressure ulcer.

\section{REFERENCES}

1. Brink P, Smith TF, Linkewich B. Factors associated with pressure ulcers in palliative home care. J Palliat Med 2006; 9(6): 1369-1375.

2. Riordan J, Voegeli D. Prevention and treatment of pressure ulcers. Br J Nurs 2009; 18(20): 20-27.

3. Yim MJ, Park HS. Study on the Pressure Ulcers in Neurological Patients in Intensive Care Units. J Korean Acad Fundam Nurs 2006; 13(2): 190-199.

4. Gallaghera P, Barrya P, Hartiganb I, Mccluskeyc P, Oconnord KM, Oconnora M. Prevalence of pressure ulcers in three university teaching hospitals in Ireland. J Tissue Viability 2008; 17(4): 103-109.

5. Kwong E, Pang SM, Aboo GH, Law SS. Pressure ulcer development in older residents in nursing home: influencing factors. J Adv Nurs 2009; 65(12): 2608-2620.

6. Thomas DR. Are all pressure ulcers avoidable?. J Am Med Dir Assoc 2003; 4(2): 43-48.

7. Adunsky A. Ohry A. Decubitus direct current treatment(DDCT) of pressure ulcers: results of a randomized double-blinded placebo controlled study. Arch Gerotol Geritr 2005; 41(3): 261-269.

8. Kloth LC, Feedar JA. Acceleration of wound healing with high voltage, monophasic, pulsed current. Phys Ther 1988; 68(4): 503-508.

9. Brown M, Gogia PP. Effects of high voltage stimulation on cutaneous wound healing in rabbits. Phys Ther 1987; 67(5): 662-667.

10. Brown M, Mcdonnell MK, Menton DN. Polarity effects on wound healing using electric stimultaion in rabbits. Arch Phys Med Rehabil 1989; 70(8): 624-627.

11. Griffin JW, Tooms RE, Mendius RA, Clifft JK, Vander ZR, El ZF. Efficacy of high voltage pulsed current for healing of pressure ulcers in patients with spinal cord injury. Phys Ther 1991; 71(6): 443-442. 
12. Recio AC, Felter CE, Schneider AC, McDonald JW. High-voltage electrical stimulation for the management of stage III and IV pressure ulcers among adults with spinal cord injury: demonstration of its utility for recalcitrant wounds below the level of injury. J Spinal Cord Med 2012; 35(1): 58-63.

13. Lee HM, Chun SH, Kang JH, Bang HS, Kim JS. Pressure ulcers treatment in physical therapy. J Korean Soc Phys Med 2008; 3(1): 67-77.

14. Gogia PP. Physical therapy modalities for wound management. J Wound Ostomy 1996; 42(1): 46.

15. Pai DR, Madan SS. Techniques in chronic wound management: review of the literature and recent concepts. J Nov Physiother 2013; 3(2): 1-7.

16. Ferrell BA. The sessing scale for assessment of pressure ulcer healing. J Am Geriatr Soc 1995; 10(5): 78-81.

17. Houghton PE, Campbell KE, Fraser CH, Harris C, Keast DH, Potter PJ, et al. Electrical stimulation therapy increases rate of healing of pressure ulcers in community-dwelling people with spinal cord injury. Arch Phys Med Rehabil 2010; 91(5): 669-678.
18. Feedar JA, Kloth LC, Gentzkow GD. Chronic dermal ulcer healing enhanced with monophasic pulsed electrical stimulation. Phys Ther 1991; 71(1): 639-649.

19. Wood JM, Evans PE. Schallreuter KU, Jacobson WE, Sufit R, Newman J, Jacobson M, et al. A multicenter study on the use of pulsed lowintensity direct current for healing chronic stage II and stage III decubitus ulcers. Arch Dermatol 1993; 129(8): 999-1009.

20. Vowden K, Vowden P. Understanding exudate management and the role of exudate in the healing process. Br J Community Nurs 2003; 8(5): 413.

21. Kuffler DP. Techniques for wound healing with a focus on pressure ulcer elimination. The Open Circulation and Vascular Journal 2010; 3(8):7484.

22. Asadi MR, Torkaman G, Hedayati M, Mofid M. Role of sensory and motor intensity of electrical stimulation on fibroblastic growth factor-2 expression, inflammation, vascularization, and mechanical strength of full-thickness wounds. J Rehabil Dev 2013; 50(4): 489-498. 